\title{
Basic-level kinds and object persistence
}

\author{
Mijke Rhemtulla And D. Geoffrey Hall \\ University of British Columbia, Vancouver, British Columbia, Canada
}

\begin{abstract}
In three experiments, we explored the basis of adults' judgments of individual object persistence through transformation. Participants watched scenarios in which an object underwent a transformation into an object belonging to the same or a different basic-level kind. Participants were queried about the object's persistence through the transformation as an individual (indexed by its proper name) and as a member of the original kind (indexed by its basic-level count noun in Experiments 1 and 2, or by its superordinate-level noun in Experiment 3). In all experiments, participants rated objects that were altered in a way that maintained basic-level kind to be less likely to retain their proper name than those that were altered in a way that changed basic-level kind. These findings suggest that shared basic-level kind membership serves as a dimension of similarity over which objects' unique individual identities are highlighted. We discuss the implications of the results for existing theoretical accounts of adults' judgments of individual object persistence.
\end{abstract}

Researchers who study object concepts have recently begun to broaden the scope of their investigations to examine not only the representation of kinds (e.g., person, cat) but also the representation of individuals (e.g., Queen Elizabeth, Felix). A central goal in the study of individual object concepts is to explain the basis for attributions of persistence: What underlies intuitions that an individual encountered at one time is the same individual as one encountered earlier? There are several theoretical accounts of such object persistence in the literature, each offering a different answer to this question. Perhaps the simplest is based on similarity: Intuitions that two object appearances represent the same persisting individual are based on the perceived similarity between them. Yet although this proposal may hold intuitive appeal, a pure similarity account of the basis for intuitions about individual persistence is prima facie problematic. For example, we may judge many highly similar objects (such as identical twins) to be different individuals, whereas we may judge other highly dissimilar objects (such as a caterpillar seen at one time and a butterfly seen later) to be the same individual (for discussion, see Rips, Blok, \& Newman, 2006).

Moving beyond similarity, an alternative account of the basis for judgments of object persistence appeals to continuity, either spatiotemporal (e.g., Bloom, 2000) or causal (Blok, Newman, \& Rips, 2005; Rips et al., 2006). According to this alternative, intuitions about object persistence arise from the inference that an object observed earlier traces a connected path through space and time to an object observed later, or that the latter is a causal outgrowth of the former. Another alternative to the similarity account posits that judgments of object persistence are linked fundamentally to kind (or sortal) representations, as proposed initially by philosophers (e.g., Hirsch,
1982; Wiggins, 2001) and taken up by psychologists (e.g., Bonatti, Frot, Zangl, \& Mehler, 2002; Hall, 1998; Macnamara, 1986; Rhemtulla \& Xu, 2007; Xu, 1997; Xu \& Carey, 1996; Xu, Cote, \& Baker, 2005). According to this account, intuitions about the persistence of objects may be linked to inferences about continuity, but they also depend critically on the availability of a kind concept, typically a basic-level kind concept (e.g., cat, rather than the subordinate-level Burmese or the superordinate-level animal; see Macnamara, 1986), covering objects observed earlier and later. For example, in order for a cat seen at one time to be judged to be the same individual as one seen later, the later object must also be a cat.

To obtain evidence bearing on these accounts, several researchers have developed a paradigm that involves gathering judgments about the persistence of individual objects that undergo change, brought about by a magician (Liittschwager, 1995) or by a science-fiction machine (Blok et al., 2005). The different theoretical accounts make different predictions about persistence judgments in this paradigm. On the one hand, the spatiotemporal and causal continuity accounts predict that persistence judgments will depend on whether the pre- and postchange objects are seen as connected, by either a spatiotemporal or a causal path. The causal continuity view makes the further prediction that people will judge that objects are less likely to persist as the transformation increases in severity, because causal processes more easily produce closely related than distantly related objects (Rips et al., 2006). On the other hand, the kind/sortal account can be interpreted as predicting that judgments of individual persistence will depend on the existence of a kind concept, typically a basic-level kind concept, applicable to both pre- and postchange objects. As a result, changes that pre-

M. Rhemtulla, mijke@psych.ubc.ca 
serve kind membership will be more likely to promote judgments of persistence than those that cross the boundary between basic-level kinds.

Previous research using this paradigm has provided support for continuity accounts of individual persistence and, in particular, for causal continuity. First, adults in this research have shown a willingness to judge individual objects as persisting through a change in basic-level kind, contrary to the predictions of the kind/sortal account (Blok et al., 2005; Liittschwager, 1995; Rips et al., 2006). Second, Blok et al. found a monotonic decline in ratings of individual persistence, since objects were described as undergoing increasingly radical transformations, as predicted by the causal continuity view. Blok et al. showed adults a picture of an object (e.g., a cat) that was labeled with a proper name (e.g., "Jane"). They were then told that the object was placed into a machine and split into particles that were subsequently reassembled. The adults then rated their certainty on a $0-9$ scale that the resulting object belonged to the same kind (i.e., had the same basic-level count noun) and was the same individual (i.e., had the same proper name), with larger numbers indicating greater certainty. Blok et al. assigned participants to one of three conditions, according to the severity of the changes. For one third of the participants in what we will call the identical-looking condition, the pictured pre- and postchange objects looked identical (e.g., cat transformed into identical-looking cat); for another third in the crossbasic condition, the pre- and postchange pictures were from different basic-level kinds (e.g., cat into dog); and for the final third in the cross-domain condition, the preand postchange pictures were from kinds that crossed a domain-level kind boundary (e.g., cat into boat). The mean kind persistence ratings were straightforward: at ceiling when the kind did not change (identical-looking condition) and near floor when it did (cross-basic and crossdomain conditions). Mean individual persistence ratings were consistent with the predictions of the causal continuity account: highest (around 5.5) in the identical-looking condition, lower (around 2.2) in the cross-basic condition, and lowest (below 1) in the cross-domain condition.

Although Blok et al.'s (2005) results favor the causal continuity view over the kind/sortal view, the design of their experiment included only three degrees of transformation. Of particular note is the fact that the design omitted a condition in which objects were shown as undergoing transformations within a basic-level kind (e.g., from one cat into a different-looking cat). This omission is noteworthy for two reasons. First, there is a significant distance between the identical-looking and cross-basic conditions in terms of the severity of the change; filling in the gap by including a within-basic condition is important in order to provide a thorough test of the effect of transformational distance on persistence judgments. Second, the causal continuity account predicts that adults' certainty ratings about individual persistence in a within-basic condition will be higher than will those in the cross-basic condition. Commenting on the distinction between a transformation that crosses or does not cross a basic-level kind boundary,
Rips et al. (2006) wrote, "[T]he category distinction is correlated with a variation in causal distance." Making specific reference to the transformation of a table, the authors wrote, "It is easier for causal factors to produce from the original table appearance a second table appearance than a chair appearance" (p. 6).

The impetus for the present research was simple: to conduct a new object transformation experiment, similar in design to that of Blok et al. (2005) but incorporating a within-basic condition. In Experiment 1, participants watched object transformation scenarios similar to those presented in Blok et al.'s experiment. Participants then rated both their certainty that a prechange object's kind label (count noun) continued to apply to a postchange object (kind persistence rating), and their certainty that the prechange object's proper name still applied (individual persistence rating). We varied between participants the severity of the transformation. As did Blok et al., we included cross-basic and cross-domain conditions, to which we added a within-basic condition.

We state here at the outset the striking primary result of Experiment 1: Individual persistence ratings were significantly lower in the within-basic than in the cross-basic condition, directly counter to the predictions of the causal continuity account. This discovery led us to conduct Experiments 2 and 3, which were modified partial replications and extensions of Experiment 1. In both of these follow-up experiments, we obtained the same counterintuitive result that we did in the first experiment. In the General Discussion, we take up all the findings, offer an interpretation, and discuss their implications for theories of individual persistence.

\section{EXPERIMENT 1}

\section{Method}

Participants and Design. One-hundred twenty adults recruited from undergraduate psychology classes received course credit for participating. Twenty-three males and 92 females participated; gender data were not gathered for 5 participants. Participants were randomly assigned to one of six conditions, defined by crossing two domains with three levels of transformational distance. Domain refers to the domain of the prechange objects (animal or artifact). Previous research has documented a significant effect of this variable on individual persistence judgments (e.g., Hall, 1998). Transformational distance refers to whether the object transformations were within the basic level (within-basic; e.g., a duck turned into a differentlooking duck), across the basic level but within the superordinate level (cross-basic; e.g., a duck turned into a cat), or across domains (cross-domain; e.g., a duck turned into a helmet).

Materials and Procedure. Participants sat individually at computers. They were first given a blank answer sheets and then shown a drawing of a machine called an "atom reassembler." Based on the transformation device described in Blok et al. (2005), this machine consisted of two compartments connected by a hose; one compartment was labeled "disintegrater" and the other "reassembler." Participants read:

An atom reassembler is a machine that transforms objects by disintegrating them into all of their atoms, moving the atoms through a small hose, and recombining those atoms in a different way. You put an object into the disintegrater, tell the computer what outcome you want, and watch it work. 


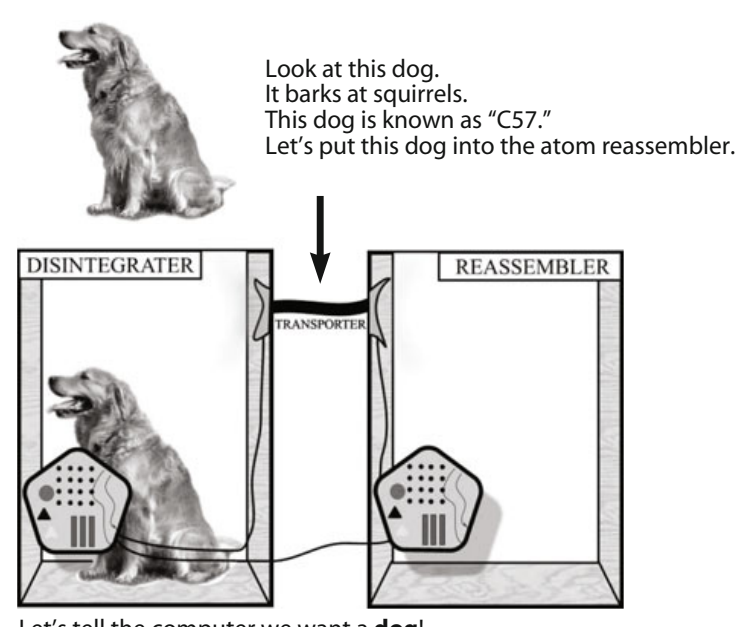

Let's tell the computer we want a dog!

One moment please, while the machine works.

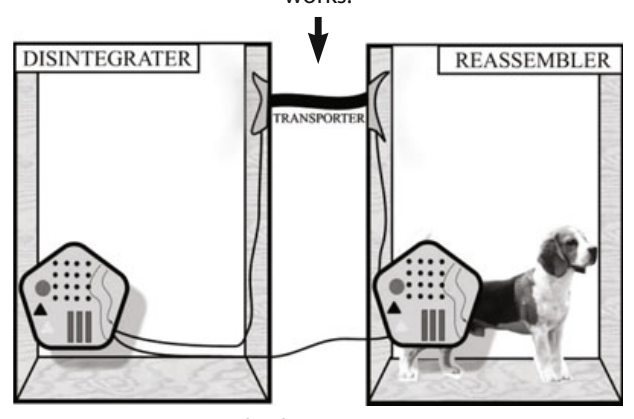

Look what came out!

Figure 1. Sample trial from Experiment 1, within-basic condition.

In all conditions, participants, answering questions on the answer sheet as they progressed, watched a slide show depicting 10 object transformations, the order of which was counterbalanced across participants. For each transformation, participants first saw a slide showing the prechange object, accompanied by a description that included the object's basic-level kind label and its proper name. For example, when the initial object was a duck, the slide read, "Look at this duck. It swims after floating breadcrumbs. This duck is known as 'V74.' Let's put this duck in the atom reassembler.' Participants then saw a slide showing the object entered in the "disintegrater" compartment of the machine, accompanied by the text, "Let's tell the computer we want a [basic-level kind label for the postchange object]." The screen displayed bright colors for several seconds while the machine "worked," and then a slide appeared showing the machine with the postchange object located in the "reassembler" compartment, accompanied by the phrase, "Look what came out!" Finally, participants saw a slide with two test questions, presented in a counterbalanced order between participants. Instructions read, "Please answer the following questions on your answer sheet regarding the object in the reassembler. Please answer on a scale of 1-7, where 1 is 'definitely no' and 7 is 'definitely yes."' The individual persistence question queried the identity of the named individual (e.g., "Is this still V74?"), and the kind persistence question queried the identity of the basic-level kind to which the original individual belonged (e.g., "Is this still a duck?"). Participants recorded their answers on the answer sheet, by circling a number from 1 to 7 for each question.

To depict the pre- and postchange objects, we used a set of 40 photographs, including two exemplars of each of 10 basic-level animal kinds and 10 basic-level artifact kinds. One set of 20 always served as prechange photographs, including one exemplar of each of the 20 basic-level kinds (10 animals and 10 artifacts). The other set of 20 always served as postchange photographs. The postchange photographs were paired differently with the prechange photographs, according to condition. In the within-basic conditions, the postchange photograph showed another object from the same basic-level kind as the prechange object. In the cross-basic conditions, the postchange photograph showed an object from a different basic-level kind within the same domain (i.e., animal or artifact) as the prechange object. In the cross-domain conditions, the postchange photograph showed an object from a different domain than that of the prechange object. We asked half the participants in each condition, as they completed the task, to provide explanations for their responses to the questions posed at the end of each transformation. We asked them to turn over their answer sheet and write brief explanations why they answered the questions the way they did. Participants typically took $10-15$ min to complete the task.

To assess the perceived similarity of the pre- and postchange photographs in each condition, we gave 10 participants who did not take part in the experiment proper all the pairs of objects from each condition, in one of two random orders, and asked them to rate their similarity on a scale from 1 to 7 , where 1 was highly dissimilar and 7 was highly similar. For the within-basic condition pairs, the mean similarity rating was $5.73(S D=0.89)$; for the cross-basic condition pairs, the mean was $2.22(S D=0.75)$; and for the crossdomain condition pairs, the mean was $1.32(S D=0.57)$. A repeated measures ANOVA of the mean similarity ratings from each condition was highly significant $\left[F(2,18)=107.44, p<.001, \eta_{\mathrm{p}}^{2}=.92\right]$. Follow-up pairwise comparisons using a Bonferroni correction were also highly significant (all $p \mathrm{~s}<.005$ ). These findings reveal that the perceived similarity of the pre- and postchange objects declined monotonically as the transformational distance increased.

\section{Results}

As in Blok et al. (2005), ratings of kind persistence were straightforward. When the postchange object maintained its basic-level kind, the mean kind persistence rating was near ceiling; when it changed kinds, it was near floor. A two-way (domain $\times$ transformational distance) between-subjects ANOVA using the mean kind persistence ratings yielded a main effect of transformational distance $\left[F(2,114)=1,677.82, p<.001, \eta_{\mathrm{p}}^{2}=.97\right] . .^{1}$ Tukey post hoc tests confirmed that the mean kind persistence rating in the within-basic conditions was significantly higher than it was in both the cross-basic and the crossdomain conditions. Table 1 lists condition means and standard deviations for all three experiments.

Individual persistence ratings presented a very different pattern of results. In the cross-domain conditions, representing the most radical change, the mean individual persistence rating was 2.71 on our 7-point scale. This value was significantly lower than the midpoint of the scale (i.e., 4) $[t(39)=-3.77, p<.005$, two-tailed], compared with a Bonferroni-adjusted alpha level of .02. In the less radical cross-basic conditions, the mean individual persistence rating was 3.82 , higher than the mean in the cross-domain conditions and not different from the scale midpoint $[t(39)=-0.47, p>.5$, two-tailed]. In the within-basic conditions, involving the mildest trans- 
Table 1

Means and Standard Deviations (SDs) of Individual- and Kind-Persistence Ratings

\begin{tabular}{llllll}
\hline & \multicolumn{2}{c}{ Individual } & & \multicolumn{2}{c}{ Kind } \\
\cline { 5 - 6 } & $M$ & $S D$ & & $M$ & $S D$ \\
\hline Experiment 1 & & & & \\
$\quad$ Within-basic condition & 1.91 & 1.24 & & 6.71 & 0.52 \\
$\quad$ Cross-basic condition & 3.82 & 2.45 & & 1.20 & 0.64 \\
$\quad$ Cross-domain condition & 2.71 & 2.16 & & 1.09 & 0.23 \\
Experiment 2 & & & & & \\
$\quad$ Identical-looking condition & 5.08 & 2.27 & & 6.78 & 0.71 \\
$\quad$ Within-subordinate condition & 2.46 & 1.25 & & 6.69 & 1.19 \\
$\quad$ Within-basic condition & 3.05 & 2.38 & & 6.84 & 0.41 \\
$\quad$ Cross-basic condition & 5.39 & 1.95 & & 1.68 & 1.69 \\
Experiment 3 & & & & \\
$\quad$ Within-basic condition & 2.94 & 2.40 & & 6.78 & 0.68 \\
$\quad$ Cross-basic condition & 4.46 & 2.37 & & 6.49 & 0.90 \\
\hline
\end{tabular}

Note $-n=20$ per condition in each experiment. Rating scale: $1=$ definitely no; 7 = definitely yes.

formation, the mean individual persistence rating should have been higher than the mean in the cross-basic conditions, according to the causal continuity account. However, the mean rating in these conditions was lowest of all three transformational distance conditions, just 1.91 on the 7-point scale. This value was significantly lower than the midpoint of the scale $[t(39)=-10.61, p<.001$, two-tailed].

A three-way [domain $\times$ transformational distance $\times$ explanations elicited (yes, no)] between-subjects ANOVA, using the mean individual persistence ratings, revealed a significant main effect of transformational distance $\left[F(2,114)=8.92, p<.001, \eta_{\mathrm{p}}^{2}=.14\right]$. Tukey post hoc tests confirmed that the mean in the cross-basic conditions was significantly higher than the mean in the crossdomain conditions; that the mean in the within-basic conditions was significantly lower than the mean in the cross-basic conditions; and that the difference between the means in the within-basic and cross-domain conditions was not significant. There was no significant main effect of domain or of explanations elicited, and there were no significant two-way interactions. ${ }^{2}$

Explanations. Recall that we obtained written explanations after each transformation from half the participants $(n=20)$ in each transformational distance condition. Participants provided explanations for both their kindpersistence and their individual-persistence ratings, but we coded only the content that pertained to the individualpersistence ratings.

After reading the written explanations, we noticed a striking tendency for participants to comment on the differences between the pre- and postchange objects in the within-basic conditions but not in the other conditions. To explore this tendency, we asked two independent coders to classify the 60 explanations as falling into one of four categories. The first category was differences $(n=19)$, and it was assigned if the explanations for the individual persistence ratings invoked contrasts between the two objects. Sample explanations were "Its specific appearance (color, texture) is widely different from the original";
"Color and pattern of shoe are different"; and "Color and figure difference." The second category was similarities $(n=9)$, and it was assigned if the explanations alluded to properties shared by the pre- and postchange objects. Sample explanations were "They are the same atoms"; "It consists of the same atoms"; and "It has its atoms." The third category, both $(n=19)$, was assigned if the explanations alluded to both differences and similarities; the fourth category, neither $(n=13)$, was assigned if they referenced neither. The proportion of agreement between coders was .83, and Kappa was .78, a value that Landis and Koch (1977) describe as "substantial." Disagreements were resolved through discussion.

The primary finding was that participants in the withinbasic conditions were most likely to provide differences explanations, and they strongly favored these explanations over similarities explanations. To analyze the explanation results, we first compared the number of differences explanations across transformational distance conditions. We carried out chi-square tests, comparing the number of differences explanations in the within-basic conditions ( $n=14)$ with the number in the cross-basic $(n=3)$ and with the number in the cross-domain $(n=2)$ conditions. Both tests were significant $\left[\chi^{2}(1, N=40)=12.38, p<\right.$ $.001, \varphi=.56$, and $\chi^{2}(1, N=40)=15.00, p<.001, \varphi=$ .61 , respectively]. The difference between the numbers in the cross-basic and cross-domain conditions was not significant $\left[\chi^{2}(1, N=40)=0.23, p>.50, \varphi=.08\right]$. Second, we used the binomial distribution to compare the number of differences explanations with the number of similarities explanations within each transformational distance condition. In the within-basic conditions, there were significantly more differences $(n=14)$ than similarities $(n=1)$ explanations $(p<.001)$. In the two other transformational distance conditions, the difference between the number of differences and similarities explanations was not significant (cross-basic, $n=3$ and $n=4$, respectively; crossdomain, $n=2$ and $n=4$, respectively).

\section{Discussion}

Previous results using this transformation paradigm (Blok et al., 2005; see also Liittschwager, 1995) have revealed a monotonic decline in individual persistence ratings with increasing transformational distance. Those findings have been interpreted as favoring the causal continuity account of individual persistence, which posits that it is easier for a given causal process to produce more closely related than more distantly related objects (Rips et al., 2006). The findings have also been viewed as contradicting the sortal account, which predicts that participants should not judge an object to persist as the same individual if it undergoes a change in (basic-level) kind. The present results provide further evidence against the sortal account, insofar as adults often judged an individual to persist through a change in kind. Contrary to the predictions of causal continuity, however, the present results revealed that individual persistence ratings were significantly lower for a less severe change in the within-basic conditions (e.g., duck into different-looking duck) than for 
a more severe change in the cross-basic conditions (e.g., duck into cat). In fact, individual persistence ratings in the within-basic conditions were even lower than those in the cross-domain conditions (e.g., duck into helmet). We also found no effect of domain on individual persistence ratings; whether the transformations included animals or artifacts made no difference for persistence ratings.

In explaining their individual persistence ratings, more participants alluded to differences between the pre- and postchange objects in the within-basic conditions than in either of the other transformational distance conditions. Furthermore, in the within-basic conditions, more participants made reference to differences than to similarities; in the other transformational distance conditions, equivalent numbers made reference to differences and to similarities. These findings suggest that the contrasts between the pre- and postchange objects were especially salient in the within-basic conditions, despite the fact that these were the conditions in which the objects were rated most similar.

In the General Discussion, we will offer an interpretation and discussion of these counterintuitive results. First, however, we present two more experiments, in which we sought to replicate and extend the primary findings of Experiment 1 . Experiment 2 involved several changes to the method of Experiment 1. Most substantially, we elaborated the within-basic conditions of Experiment 1 into three more specific conditions. In Experiment 1, the preand postchange photographs used for each transformation in the within-basic conditions always showed different objects from within the same basic-level kind, but we did not systematically vary whether the pairs came from within the same or different subordinate-level kinds. In Experiment 2, the within-basic condition always included two exemplars of animals that belonged to different subordinate-level kinds within the same basic-level kind (e.g., a mallard duck transformed into a white duck). We now also included a within-subordinate condition (e.g., a mallard duck transformed into a different-looking mallard duck) in order to examine individual persistence ratings following a narrower kind change within the basic level. In addition, we included an identical-looking condition, as did Blok et al. (2005), in which the postchange object looked identical to the prechange object (e.g., a mallard duck transformed into an identical-looking mallard duck). Our reason for including the identical-looking condition was to determine whether we could replicate the results from the entire range of conditions in Blok et al. while maintaining the counterintuitive finding from the withinbasic condition.

In this experiment, the causal continuity account again straightforwardly predicts that individual persistence ratings will rise as the transformation becomes less extreme, resulting in an increase in mean rating from the crossbasic, to the within-basic, to the within-subordinate, to the identical-looking condition. If, however, the withinbasic condition result of Experiment 1 is replicable, then individual persistence ratings will again be lower in the within-basic than in the cross-basic condition. Further- more, if we replicate the within-basic condition finding, then the results from the two new conditions will reveal whether the result from that condition also emerges in a condition involving a more restricted change within the basic level (within-subordinate condition) and/or in a condition involving no change at all (identical-looking condition).

\section{EXPERIMENT 2}

\section{Method}

Participants and Design. Eighty adults from undergraduate psychology classes received course credit for participating. None had participated in Experiment 1. Fifteen males and 62 females participated; gender data were not collected for 3 participants. Participants were randomly assigned to one of four conditions, defined according to level of transformational distance. As in Experiment 1, we included a cross-basic and a within-basic condition. To these, we added a within-subordinate condition and an identical-looking condition. Because we observed no effects involving domain in Experiment 1 , we did not manipulate domain in this experiment; all objects were animals. We also did not include a cross-domain condition, because here our focus was on the relation between the results in the cross-basic, the within-basic, and the two new conditions.

Materials and Procedure. The procedure was the same as in Experiment 1, with three exceptions. First, because we did not include artifacts, we named the animals using proper names and a sentence frame more appropriate for animals. Instead of alphanumeric strings, we used nonsense syllables that were introduced using a more natural locution for naming animals: "This [basic-level count noun]'s name is [nonsense syllable]" (e.g., "This duck's name is Maf"). Second, after each transformation, we showed participants a slide showing the prechange object on the left, next to a large red arrow pointing to the postchange object on the right. At the bottom of the slide, participants read "Here is a review of what you just saw." This slide was included as a reminder of what the prechange animal looked like. We made this addition because the pre- and postchange objects looked very similar, or the same, in the within-basic, withinsubordinate, and identical-looking conditions, and we wanted to ensure that participants encoded the objects appropriately. Third, we did not obtain explanation data.

To depict the pre- and postchange objects, we used 30 photographs, many different from those used in Experiment 1. Ten of these always served as the prechange photographs, and they depicted exemplars of 10 different basic-level animal kinds. Seven of these 10 basic-level kinds were the same kinds we used in Experiment 1. The remaining 20 served as postchange photographs. Ten of them showed exemplars of different animals from the same subordinatelevel kinds as shown in the prechange photographs; and 10 showed exemplars of animals from different subordinate-level kinds within the same basic-level kinds as the prechange objects. In the identicallooking condition, the postchange photographs were simply copies of the prechange photographs. In the within-subordinate condition, the postchange photographs showed different animals from within the same subordinate-level kinds. In the within-basic condition, the postchange photographs showed animals from different subordinatelevel kinds within the same basic-level kinds. In the cross-basic condition, the postchange photographs showed animals from different basic-level kinds; these were the same photographs we used in the within-subordinate condition.

As in Experiment 1, we assessed the perceived similarity of the pre- and postchange photographs in each condition. We gave 16 participants who did not take part in either Experiment 1 or 2 all the pairs of objects from each condition (in one of two random orders) and asked them to rate their similarity on a scale from 1 to 7 , as in Experiment 1. For the identical-looking condition pairs, the mean similarity rating was $6.95(S D=0.20)$; for the within-subordinate 
condition pairs, the mean was $5.32(S D=0.71)$; for the within-basic condition pairs, the mean was $4.50(S D=0.86)$; and for the crossbasic condition pairs, the mean was $1.80(S D=0.61)$. A repeated measures ANOVA of the mean similarity ratings from the four conditions was highly significant $\left[F(3,45)=223.44, p<.001, \eta_{\mathrm{p}}^{2}=\right.$ .94]. Follow-up pairwise comparisons using a Bonferroni correction were also highly significant (all $p \mathrm{~s}<.001$ ). These findings again indicate that the perceived similarity of the pre- and postchange objects fell monotonically as transformational distance increased.

\section{Results}

As in Experiment 1, kind persistence ratings were uncomplicated. The means in the three conditions where basic-level kind was unchanged (within-basic, withinsubordinate, identical-looking) were near ceiling, and the mean in the cross-basic transformation condition was near floor. A one-way ANOVA using the mean kind persistence ratings yielded a significant effect $[F(3,76)=158.40$, $\left.p<.001, \eta_{\mathrm{p}}^{2}=.86\right]$. Tukey pairwise comparisons confirmed that the mean kind persistence rating in the crossbasic condition was significantly lower than the mean in each of the three conditions where basic-level kind was maintained.

Turning to the individual persistence ratings, we replicated the striking main result from Experiment 1: The mean was substantially lower in the within-basic condition $(M=3.05)$ than in the cross-basic condition $(M=5.39)$. In addition, the mean in the within-subordinate condition $(M=2.46)$ was low and similar in value to the mean in the within-basic condition. In the identical-looking condition $(M=5.08)$, however, the mean was relatively high. A one-way ANOVA using the mean individual persistence ratings revealed a significant effect $[F(3,76)=10.48$, $\left.p<.001, \eta_{\mathrm{p}}^{2}=.29\right]$. Tukey post hoc tests confirmed that the mean ratings in the within-subordinate and withinbasic conditions were significantly lower than those in the identical-looking and cross-basic conditions. Furthermore, the mean rating in the within-basic condition fell marginally below the scale midpoint $[t(19)=-1.79, p=$ .04 , one-tailed], compared with an adjusted alpha level of .02 , and the within-subordinate condition mean fell significantly below it $[t(19)=-5.50, p<.001$, two-tailed $] .^{3}$ In contrast, the identical-looking condition mean fell marginally above the scale midpoint $[t(19)=2.12 ; p=.05$, two-tailed], and the cross-basic condition mean was significantly above it $[t(19)=3.18, p<.005$, two-tailed].

\section{Discussion}

The results of Experiment 2 replicated and extended those of Experiment 1. As in Experiment 1, participants' ratings of individual persistence were lower when they judged a transformation that involved a within-basic change than when they judged one that involved a crossbasic change.

The difference between the mean individual persistence rating in the within-basic and cross-basic conditions was similar in this experiment to what it was in Experiment 1, although the mean values in both conditions were more than one point higher in this experiment. Given that there were several methodological differences between the two experiments, it is difficult to pinpoint the reason for the higher means in Experiment 2. One possible explanation is that it reflects the impact of the reminder slide that was added before participants provided their ratings for each transformation. Recall that this reminder slide showed an arrow pointing from the prechange object to the postchange one; it is plausible that the presence of this arrow encouraged participants to infer a connection between the two object appearances, resulting in higher mean ratings in both conditions.

There were also two new findings. First, the mean individual persistence rating in the within-subordinate condition was similar to, and slightly lower than, the mean in the within-basic condition. The "within-basic" effect we have documented thus seems to arise even when participants see pre- and postchange objects from within a more restricted kind within the basic level (e.g., two different-looking poodles, both from the same basiclevel kind, $\operatorname{dog}$ ). It is striking that the mean rating was even lower in the within-subordinate condition than it was in the within-basic condition, given that the withinsubordinate condition objects were judged to be more similar than those in the within-basic condition. Second, the mean individual persistence rating in the condition involving identical-looking pre- and postchange objects (i.e., no visible change) was higher than that in the conditions involving within-basic or within-subordinate changes, and the mean was similar to the mean in the condition in which the transformation crossed the basic-level boundary. This result indicates that the low mean ratings we observed in the within-basic and within-subordinate conditions depended on the presence of a perceptible contrast between pre- and postchange objects from within the same basiclevel kind.

Now we report one final experiment. Experiment 3 was a modified partial replication of Experiment 2, designed to assess the role of basic-level kind labels in producing the within-basic effect documented in Experiments 1 and 2. In Experiments 1 and 2, recall that every object was introduced with a basic-level count noun, each transformation was described using basic-level count nouns, and kind persistence ratings were always elicited for the basic-level kind. These facts raise the possibility that the within-basic effect depends on the explicit use of basiclevel count nouns to describe the objects in the task. To address this issue, we ran new versions of the within-basic and cross-basic conditions from Experiment 2, replacing all basic-level count nouns with the superordinate-level count noun "animal." If participants' ratings of individual persistence in Experiments 1 and 2 were independent of the explicit use of basic-level count nouns, then we expected to obtain findings in the two conditions of this experiment similar to those we obtained in the corresponding conditions of the first two experiments.

\section{EXPERIMENT 3}

\section{Method}

Participants and Design. Forty adults from undergraduate psychology classes received course credit for participating. Nineteen males and 18 females participated; gender data were not collected 
for 3 participants. Participants were randomly assigned to either the within-basic or the cross-basic condition.

Materials and Procedure. The materials and procedure were the same as those used in the within-basic and cross-basic conditions in Experiment 2, except that we replaced all basic-level count nouns with the superordinate-level count noun "animal," including the label used in the kind-persistence question. For example, for the duck transformation, we used the word "animal" wherever we had used the word "duck" in Experiment 2. Participants read, "Look at this animal. It swims after floating breadcrumbs. This animal's name is Maf. Let's put this animal in the atom reassembler," and on the following slide, "Let's tell the computer we want an animal." The kind-persistence question read, "Is this still an animal?"

\section{Results}

As in previous experiments, kind-persistence ratings were clear cut. In both the within-basic and cross-basic conditions, the mean ratings were near ceiling. This was an unsurprising result, given that we now queried the persistence of the superordinate-level kind (i.e., "Is this still an animal?"), and superordinate-level kind was maintained through the transformation in both conditions. The difference between mean ratings in the two conditions was not significant $[t(38)=1.23, p>.20, d=.39]$.

We then turned to the individual persistence ratings in order to determine whether the within-basic effect observed in Experiments 1 and 2 remained now that participants did not read basic-level count nouns during the task. It did. As in Experiments 1 and 2, the mean individual persistence rating was significantly lower in the within-basic condition $(M=2.94)$ than in the cross-basic condition $(M=4.46)[t(38)=2.02, p=.03$, one-tailed, $d=.64]$. Moreover, the cross-basic condition mean was no different from the scale midpoint $[t(19)=0.86, p>$ .25 , two-tailed], compared with an adjusted alpha level of .03 ; however, the within-basic condition mean was significantly lower than the midpoint $[t(19)=-1.99 ; p=$ .03 , one-tailed].

\section{Discussion}

Experiment 3 provided another replication of the finding that individual persistence ratings were lower when participants judged a transformation that involved a within-basic change than when they judged one that involved a crossbasic change. This result emerged despite the fact that we did not mention basic-level count nouns in describing the objects in either condition, despite the fact that participants in both conditions heard the same superordinate-level count noun ("animal") to describe the objects, and despite the fact that participants in both conditions rated kind persistence at the superordinate level. This replication of the within-basic effect reveals that the effect is not dependent on the use of basic-level count nouns.

\section{GENERAL DISCUSSION}

In three experiments, adults rated kind persistence (i.e., maintenance of a count noun label) and individual persistence (i.e., maintenance of a proper name) in a paradigm in which an object was transformed by a machine that reduced it to its constituent atoms, transferred the atoms to a new location, and then reassembled the atoms. In a prior experiment using this paradigm (Blok et al., 2005), a key result was that ratings of individual persistence declined monotonically as the transformation grew in severity, from a change into an identical-looking object to a change into an object from a different basic-level kind to a change into an object from a different ontological domain. The causal continuity theory predicted this transformational distance effect, on the grounds that intuitions of individual persistence are based on inferences that objects seen later are causal outgrowths of ones seen earlier, and that a given causal process should produce closely related objects more easily than distantly related ones (Rips et al., 2006). Blok et al. did not, however, include a full range of possible transformational distances in their design and, in particular, omitted a condition involving transformations within a basic-level kind. According to the causal continuity account, individual persistence ratings should be higher following within-basic than following cross-basic changes.

In our experiments, we added a within-basic condition to a design similar in other respects to that of Blok et al. (2005). Our counterintuitive finding, obtained in all three experiments, was that participants gave significantly lower ratings of individual persistence to an object that experienced a within-basic change than to an object that experienced a cross-basic change. Moreover, we observed the effect when participants reasoned about animals or artifacts (Experiment 1); when the transformation was between subordinate-level kinds within the basic level or within a subordinate-level kind (Experiment 2); and when the objects involved in the transformation were described using a superordinate-level count noun rather than a basic-level count noun (Experiment 3). The finding did not, however, obtain when the transformation involved identical-looking objects, suggesting that it depended on the existence of a perceptible contrast between pre- and postchange objects.

As we have noted, our findings run counter to the predictions of the causal continuity theory (Rips et al., 2006), but the results are not easily explained under other existing theoretical accounts of individual persistence, either. For instance, the spatiotemporal continuity account (e.g., Bloom, 2000) makes no predictions about differences in individual persistence ratings across transformations of increasing severity, because these ratings should simply reflect whether there is a spatiotemporal path connecting the pre- and postchange objects. In all conditions of the present experiments, there was such a connection (via the transferred atoms), yet the mean individual persistence ratings differed significantly across conditions. Moreover, the kind/sortal account can be read as predicting that ratings of individual persistence should be lower for transformations that cross a (basic-level) kind boundary than for those that remain within it. Yet the mean ratings showed exactly the opposite pattern.

Our interpretation of the results appeals to insights from the literature on the role of similarity in comparison processing. A counterintuitive finding in that literature is that people find it easier to detect certain differences between two objects when the objects under comparison are similar than when they are dissimilar; for example, people 
list more differences between two similar objects such as a sneaker and a sandal than between two dissimilar objects such as a sneaker and a paper clip (Gentner \& Markman, 1994; see also Gentner \& Markman, 1997; Gentner \& Medina, 1998; Gentner \& Namy, 2006; Markman \& Gentner, 1993; Markman \& Wisniewski, 1997; Medin, Goldstone, $\&$ Gentner, 1993). To interpret this effect, researchers in this area have proposed that the act of comparing two objects involves an attempt to align structured representations; when this effort succeeds, the comparison results in the highlighting of both similarities and differences between the objects. In a comparison such as sneakersandal, the two objects are similar, both conceptually (because they share basic-level kind membership) and perceptually (because they typically share many properties, notably a common overall shape). As a result, people find it easy to align representations of the objects in terms of a common structure (e.g., the shared basic-level kind and/ or shape). The comparison thus serves to highlight many so-called alignable differences between the objects; furthermore, these differences are likely to pertain to the objects' specific features (e.g., one has laces, one has straps; one covers the toes, one does not) (Gentner \& Markman, 1994). In a comparison like sneaker-paper clip, however, the two objects are dissimilar because they share neither basic-level kind membership nor shape. People thus find it harder to align representations of the objects in terms of a common structure, so they find fewer alignable differences. Moreover, the differences they do find tend to pertain to more general features of the objects, such as their functions or kind membership (Gentner \& Markman, 1994). (For related evidence from the study of word learning, see Gentner, Loewenstein, \& Hung, 2007; Klibanoff \& Waxman, 2000; Manders \& Hall, 2002; Waxman \& Klibanoff, 2000).

We propose that the preceding insights are relevant to understanding the counterintuitive findings from our experiments. Consider first that we observed lower individual persistence ratings in the within-basic condition than in the cross-basic condition. In the within-basic condition, participants faced drawing a comparison between pre- and postchange objects from the same basic-level kind. This comparison of similar objects should have led to easy alignment in terms of a common structure and therefore highlighted many alignable differences between the objects, differences that often related to their idiosyncratic properties. We suggest that by highlighting these differences between the objects' features, the within-basic comparison had the effect of heightening participants' tendency to see the objects as two distinct individuals. As a result, participants gave low ratings of individual persistence across the change. In the cross-basic condition, however, participants compared objects from different basic-level kinds. This comparison of dissimilar objects should not have led to easy alignment in terms of a common structure or, as a consequence, to the highlighting of many alignable differences between the objects. Furthermore, those alignable differences that were highlighted should have tended to relate to the objects' general kind membership, not to their specific properties. We propose that there was thus little heightening of the tendency for participants to view the objects as two distinct individuals. As a result, ratings of individual persistence were higher in this condition.

We have argued that the comparisons in the within-basic condition of our experiments highlighted many alignable differences between the pre- and postchange objects, differences pertaining to the objects' idiosyncratic properties. The result, we have proposed, was a tendency for participants to see the objects as distinct individuals and to provide low ratings of individual persistence. We have, however, also noted that such comparisons lead to the highlighting of many commonalities between the pre- and postchange objects. Why did participants not give more weight to these highlighted commonalities, using them as grounds for inferring individual persistence through the change and for giving high individual persistence ratings? The answer, we suggest, is simply that the task of rating individual persistence (e.g., answering "Is this still Maf?") invited participants to focus on the postchange object as a unique individual and, therefore, to attend to those idiosyncratic features that differentiated it from the prechange object, not to those features that it shared with the prechange object. For this reason, we believe, the alignable differences that were highlighted in the withinbasic condition received heavier weighting than the commonalities in individual persistence ratings.

The findings from the literature on similarity in comparison processing also help to make sense of our other findings. For example, the individual persistence ratings in the within-subordinate condition were even lower than those in the within-basic condition. Given the high degree of conceptual and perceptual similarity between preand postchange objects in that condition, it follows that it would have been even easier to align the objects in terms of a common structure, and alignable differences pertaining to the objects' specific features would have been highlighted to an even greater extent than in the within-basic condition. Furthermore, the relatively high individual persistence ratings in the identical-looking condition follow from the absence of alignable differences between the pairs of same-looking objects in that condition. Moreover, participants' tendency to refer to contrasts between the pre- and postchange objects in their explanations in the within-basic condition of Experiment 1 follows from the fact that many alignable differences between the objects would have been highlighted in that condition. Finally, the absence of domain differences (i.e., between animals and artifacts) in Experiment 1 is understandable on the assumption that individual persistence ratings in this task reflected the effect of similarity in comparison processing, not domain-specific knowledge. The ratings thus were similar in the two ontological domains.

The preceding interpretation might seem puzzling, given our claim at the beginning of the article that a pure similarity account of individual persistence judgments is problematic. How do we reconcile that claim with our interpretation? To be clear: Our proposal is not that similar- 
ity is at the root of all judgments of individual persistence, nor are we proposing that our failure to find a monotonic decline in individual persistence ratings with increases in transformational distance rules out a role for causal considerations in judgments in our task. Instead, we propose that intuitions about individual persistence in our experiments reflected the effects of similarity in comparison processing because the causal implications of the transformation were unclear to participants.

Recall that the transformation in our studies (and in Blok et al., 2005) was said to have a fictitious mechanical cause (i.e., an atom reassembler) whose effects were underspecified. Our participants were told nothing about the impact of the causal mechanism on the postchange objects (e.g., whether it was life-preserving, in the case of an animal transformation), and they could have had no real-world experience with it. Because of this underspecification, we suspect that participants were uncertain about the effects of the mechanism on individual persistence through the transformation. In support of this possibility, recall that the mean individual persistence ratings in these experiments (and in Blok et al., 2005) were never near ceiling, even for the mildest transformations (i.e., in the identical-looking condition), in contrast to the mean kindpersistence ratings, which were always near the top of the scale when the object maintained kind through the transformation. Because of their uncertainty about the causal mechanism, we propose that participants based their ratings of individual persistence on other features of the transformations, in particular on the perceived similarity between the pre- and postchange objects. If this proposal is correct, it offers an explanation for the distinctive (and counterintuitive) pattern of individual persistence ratings we observed across conditions in these experiments, but it does not mean that causal factors play no role in individual persistence judgments.

The importance of the last point becomes clearer when we consider participants' ratings of individual persistence in these experiments in the broader context of people's intuitions about individual persistence following everyday transformations. Many objects in the real world experience within-basic changes without any apparent threat to intuitions about their individual persistence, as when a dog grows larger over time and people judge that it remains the same dog, or a banana ripens from an inedible green to an edible yellow, all the while being seen as the same banana. Indeed, some objects in the real world undergo cross-basic changes, apparently without being judged to lose individual persistence, as when a caterpillar becomes a butterfly or a tadpole transforms into a frog. Intuitions about individual persistence in such cases are clearly at odds with the pattern of results we observed in these experiments.

We propose that what supports persistence intuitions in many real-world cases is that people understand the cause of the transformation to be inherent in the object undergoing the change, arising from the kind to which it belongs, perhaps from its essence (see Gelman, 2003; Hall, 1998). This kind is usually, but not always, marked in language by a basic-level count noun/sortal term. Our proposal leads to the prediction that people should have clear intuitions that living things persist through certain spontaneous changes (e.g., growth, ripening, metamorphosis), because they undergo such changes by virtue of being the kinds of things they are. At the same time, this proposal does not rule out the possibility that people can learn that we conventionally treat some other types of transformations as involving persistence, as when they judge artifacts of certain kinds to survive part-replacement (e.g., a car whose tires are replaced) or disassembly-reassembly (e.g., a bicycle taken apart and put back together), although intuitions about individual persistence may be less clear in these cases than in cases of spontaneously changing living things (Hall, 1998).

Our proposal is akin to the kind/sortal account described earlier, with an important difference: We posit that kinds/sortals support clear intuitions about individual persistence through transformation, insofar as they are thought to furnish the source of objects' changes, such as the causal mechanisms that bring about these changes. When an object's transformation is not seen to follow from its being the kind of thing it is, especially when the causal implications of the change are unclear or unfamiliar, we predict that people will be less likely to judge it to persist through the change. In such cases, we expect participants to default to using the similarity between the pre- and postchange objects to guide their persistence judgments, resulting in the pattern of results documented in the present experiments. We believe that an account of individual persistence that subordinates kinds/sortals to causal considerations (e.g., the causal continuity account) will fail to explain people's clearest intuitions about individual persistence, because the causes that support these intuitions are inextricably tied to kinds.

In conclusion, we stress that our observations about the difference between the transformations in the present experiments and many real-world transformations do not diminish the significance of our findings. The project of characterizing people's concepts of individual objects involves, at least in part, determining how they reason about individual persistence through change. This includes both real and fictional changes, as well as changes having either inherent or external causes. After all, just as people can understand dogs growing larger and trees flowering in real life, so too can they understand princes being turned into frogs in fairy tales or bodies being teleported in science fiction or transmogrified in the present experiments. The main contribution of these experiments is the discovery of a previously undocumented and counterintuitive pattern of results relating judgments of individual persistence in the wake of a change caused by a fictional machine to the severity of the transformation. Our interpretation of the findings generates predictions about the range of circumstances (e.g., for what kinds of things undergoing what kinds of transformations) in which we would expect to observe the pattern of individual persistence ratings uncovered in these experiments. Testing these predictions in future research will play an important role in the further elucidation of the representation of individual object concepts. 


\section{AUTHOR NOTE}

This work was funded by an NSERC Operating Grant to D.G.H. and an NSERC postgraduate scholarship to M.R. Portions were presented at the annual meeting of the Society of Philosophy and Psychology, Wake Forest University, 2005. We thank Graham Lavender, Elaine Co, Kathy Chan, and Katie Corrigall for their contributions, and Fei Xu for helpful discussions and comments. We also thank three anonymous reviewers for valuable suggestions. Address correspondence to M. Rhemtulla, Department of Psychology, University of British Columbia, 2136 West Mall, Vancouver, BC, V6T 1Z4 Canada (e-mail: mijke@psych.ubc.ca).

\section{REFERENCES}

BloK, S., Newman, G., \& RiPs, L. J. (2005). Individuals and their concepts. In W.-K. Ahn, R. L. Goldstone, B. C. Love, A. B. Markman, \& P. Wolff (Eds.), Categorization inside and outside the lab (pp. 127149). Washington, DC: American Psychological Association.

Bloom, P. (2000). How children learn the meanings of words. Cambridge, MA: MIT Press.

Bonatti, L., Frot, E., Zangl, R., \& Mehler, J. (2002). The human first hypothesis: Identification of conspecifics and individuation of objects in the young infant. Cognitive Psychology, 44, 388-426.

Gelman, S. (2003). The essential child: Origins of essentialism in everyday thought. New York: Oxford University Press.

Gentner, D., Loewenstein, J., \& Hung, B. (2007). Comparison facilitates children's learning of names for parts. Journal of Cognition \& Development, 8, 285-307.

Gentner, D., \& Markman, A. B. (1994). Structural alignment in comparison: No difference without similarity. Psychological Science, 5, $152-158$.

Gentner, D., \& Markman, A. B. (1997). Structure mapping in analogy and similarity. American Psychologist, 52, 45-56.

Gentner, D., \& Medina, J. (1998). Similarity and the development of rules. Cognition, 65, 263-297.

Gentner, D., \& NAmY, L. L. (2006). Analogical processes in language learning. Current Directions in Psychological Science, 15, 297-301.

Glass, G. V., Peckham, P. D., \& Sanders, J. R. (1972). Consequences of failure to meet assumptions underlying the fixed effects analyses of variance and covariance. Review of Educational Research, 42, 237-288.

HALL, D. G. (1998). Continuity and the persistence of objects: When the whole is greater than the sum of the parts. Cognitive Psychology, 37, 28-59.

Hirsch, E. (1982). The concept of identity. New York: Oxford University Press.

Klibanoff, R. S., \& Waxman, S. R. (2000). Basic level object categories support the acquisition of novel adjectives: Evidence from preschool-aged children. Child Development, 71, 649-659.

Landis, J. R., \& КосH, G. G. (1977). The measurement of observer agreement for categorical data. Biometrics, 33, 159-174.

LIITTSCHWAGER, J. C. (1995). Children's reasoning about identity across transformations (Doctoral dissertation, Stanford University, 1995). Dissertation Abstracts International, 55, 4623B.

Macnamara, J. (1986). A border dispute: The place of logic in psychology. Cambridge, MA: MIT Press.

MANDERS, K., \& HaLl, D. G. (2002). Comparison, basic-level categories, and the teaching of adjectives. Journal of Child Language, 29, 923-937.

Markman, A. B., \& Gentner, D. (1993). Structural alignment during similarity comparisons. Cognitive Psychology, 25, 431-467.

Markman, A. B., \& Wisniewski, E. J. (1997). Similar and different: The differentiation of basic-level categories. Journal of Experimental Psychology: Learning, Memory, \& Cognition, 23, 54-70.

Medin, D. L., Goldstone, R. L., \& Gentner, D. (1993). Respects for similarity. Psychological Review, 100, 254-278.
Rhemtulla, M., \& XU, F. (2007). Sortal concepts and causal continuity: Comment on Rips, Blok, \& Newman (2006). Psychological Review, 114, 1087-1094.

Rips, L. J., BloK, S., \& Newman, G. (2006). Tracing the identity of objects. Psychological Review, 113, 1-30.

StILl, A. W., \& White, A. P. (1981). The approximate randomization test as an alternative to the $\mathrm{F}$ test in analysis of variance. British Journal of Mathematical \& Statistical Psychology, 34, 243-252.

WaXman, S. R., \& Klibanoff, R. S. (2000). The role of comparison in the extension of novel adjectives. Developmental Psychology, 36, 571-581.

WIGGINs, D. (2001). Sameness and substance renewed. New York: Cambridge University Press.

Xu, F. (1997). From Lot's wife to a pillar of salt: Evidence that physical object is a sortal concept. Mind \& Language, 12, 365-392.

Xu, F., \& CAREY, S. (1996). Infants' metaphysics: The case of numerical identity. Cognitive Psychology, 30, 111-153.

XU, F., Cote, M., \& BAKER, A. (2005). Labeling guides object individuation in 12-month-old infants. Psychological Science, 16, 372-377.

\section{NOTES}

1. Many participants' ratings reflected strong agreement or disagreement with the questions posed, and few ratings fell in the middle range of the scale, resulting in a violation of normality in the data. Although ANOVA is robust to departures from normality, especially with a balanced design (Glass, Peckham, \& Sanders, 1972), we also analyzed the data in all experiments using approximate randomization tests, which provide a nonparametric test of the main effects (Still \& White, 1981). We obtained precisely the same pattern of significant results in all three experiments using these tests as we did using ANOVA. Because ANOVA also allowed us to test interaction effects in Experiment 1, we report the ANOVA results in the text.

2. The only other significant effect in the ANOVA was a three-way interaction between domain, transformational distance, and explanations elicited $\left[F(2,108)=3.35, p<.05, \eta_{\mathrm{p}}^{2}=.06\right]$. This interaction reflected the following: In the within-basic condition, participants who provided explanations for their answers gave higher individual persistence ratings than those who did not when the transformations involved animals, but lower ratings when the transformations involved artifacts. This interaction pattern was reversed in the cross-basic condition. In the cross-domain condition, there was no interaction between explanations elicited and domain. In order to shed further light on this interaction, we conducted the same three-way ANOVA using participants' ratings for the first trial only as the dependent measure. Note that participants provided these ratings before offering any explanations. We again observed the same significant three-way interaction $\left[F(2,108)=3.12, p<.05, \eta_{\mathrm{p}}^{2}=.06\right]$. This finding suggests that the three-way interaction observed in the overall analysis set did not stem from the fact that half the participants actually provided explanations for their ratings. We cannot, however, rule out the possibility that the interaction reflects the fact that half the participants thought through the rationale they would be offering for their ratings. At the same time, we admit to finding it difficult to understand the link between any deliberation process and the observed interaction.

3 . In calculating the $p$ values for comparisons to chance in this experiment and in Experiment 3, we used a directional hypothesis for the within-basic condition, because we expected to replicate the result of the within-basic conditions in Experiment 1. We used a nondirectional hypothesis for the other conditions, because Experiment 1 did not establish a clear precedent for these effects.

(Manuscript received October 8, 2007; revision accepted for publication November 10, 2008.) 